\title{
Rede Mãe Paranaense: análise do índice de implementação em regionais de saúde
}

\section{Rede Mãe Paranaense: analysis of the implementation index in the regional healthcare network}

\author{
Renata Andrade Teixeira' (D), Sebastião Caldeira² (D), Ana Tereza Bittencourt Guimarães ${ }^{3}$ (D), \\ Mauren Teresa Grubisich Mendes Tacla ${ }^{4}$ (D), Rosângela Aparecida Pimenta Ferrari ${ }^{5}$ (D) \\ ${ }^{1}$ Departamento de Enfermagem, Centro de Ciências da Saúde, Universidade Estadual de Londrina (UEL) - Londrina (PR), Brasil. \\ ${ }^{2}$ Departamento de Enfermagem, Centro de Ciências da Saúde, Universidade Estadual do Oeste do Paraná (UNIOESTE) - Cascavel \\ $(P R)$, Brasil. \\ ${ }^{3}$ Departamento de Biologia, Centro de Ciências da Saúde, Universidade Estadual do Oeste do Paraná (UNIOESTE) - Cascavel (PR), \\ Brasil. \\ ${ }^{4}$ Departamento de Enfermagem, Centro de Ciências da Saúde, Universidade Estadual de Londrina (UEL) - Londrina (PR), Brasil. \\ ${ }^{5}$ Departamento de Enfermagem, Centro de Ciências da Saúde, Universidade Estadual de Londrina (UEL) - Londrina (PR), Brasil.
}

Como citar: Teixeira RA, Ferrari RAP, Guimarães ATB, Tacla MTGM, Caldeira S. Rede Mãe Paranaense: análise do índice de implementação em regionais de saúde. Cad Saúde Colet, 2021;29(2):291-300. https://doi.org/10.1590/1414462X202129020428

\section{Resumo}

Introdução: A avaliação de programas pode nortear a tomada de decisão de gestores. Objetivo: Analisar o Índice de Implementação (II) da Rede Mãe Paranaense (RMP). Método: Pesquisa quantitativa retrospectiva realizada em três Regionais de Saúde (RS) do Paraná, Brasil, totalizando 55 municípios. Construíram-se indicadores de avaliação antes (2010-2011) e depois (2012-2013) da implementação da Rede. Estabeleceram-se cinco domínios com variáveis coletadas no Sisprenatal, Sinasc, SI-PNI, SIM e informações nas RS. Para cada domínio, avaliou-se a influência das variáveis do Il (adequado e inadequado). Utilizou-se do programa XLStat2014 e aplicaram-se os testes de Shapiro-Wilk, regressão logística, análise da variância fatorial e Monte Carlo, com nível de significância $p<0,05$. Resultados: O II antes (2010-2011) e depois (2012-2013) da RMP, nas três RS, apresentou diferença estatística significativa $\left(F_{6,156}=129,14\right.$; $p=0,000)$. Em 2010, todas as RS foram estatisticamente semelhantes $(p>0,05)$, demonstrando equivalência nos municípios; em 2010-2011, o II da 10a RS foi maior que da 9a RS; em 2011-2012, os valores da $10^{\text {a }}$ e da 17a RS foram estatisticamente superiores aos da 9a RS ( $p<0,05)$; em 2012-2013, a 10a RS apresentou elevado crescimento, semelhante ao da $17^{\text {a }} \mathrm{RS}(p>0,05)$ e superior ao da $9^{\mathrm{a}} \mathrm{RS}(p<0,05)$. Conclusão: O II para avaliar o desempenho da Rede nas três RS se mostrou adequado à avaliação dos indicadores. Palavras-chave: avaliação de programas e projetos de saúde; sistemas de saúde; saúde da mulher; saúde da criança.

\begin{abstract}
Background: Program evaluation can guide managers' decision-making. Objective: Analyze the Implementation Index of the Rede Mãe Paranaense healthcare network. Method: Retrospective, quantitative study conducted in three Health Regionals (HRs) in the state of Paraná, Brazil, including 55 municipalities. Evaluation indicators were built before (2010-2011) and after (2012-2013) the implementation of the network. Five domains were established, with variables collected from SISPRENATAL, SINASC, SI-PNI, SIM, and information from HR. For each domain, the influence of the Implementation Index (II) variables (adequate vs. inadequate) was evaluated. The XLSTAT2014 ${ }^{\circ}$ software was used and the Shapiro-Wilk and Monte Carlo tests, Logistic Regression Analysis, and Factorial Analysis of Variance were applied at a $5 \%$ significance level $(p<0.05)$. Results: Statistically significant differences
\end{abstract}

Este é um artigo publicado em acesso aberto (Open Access) sob a licença Creative Commons Attribution, que permite uso, distribuição e reprodução em qualquer meio, sem restrições desde que o trabalho original seja corretamente citado. 
$\left(F_{6.156}=129.14 ; p=0.000\right)$ were observed between the II values before (2010-2011) and after (2012-2013) the implementation of the Rede Mãe Paranaense healthcare network in the three HRs. In 2010, all HRs were statistically similar ( $p>0.05$ ), demonstrating equivalence in the municipalities; from 2010 to 2011, the II of the $10^{\text {th }} \mathrm{HR}$ was higher than that of the $9^{\text {th }} \mathrm{HR}$; from 2011 to 2012 , the $10^{\text {th }}$ and $17^{\text {th }} \mathrm{HRs}$ were statistically superior to the $9^{\text {th }} \mathrm{HR}(p<0.05)$; from 2012 to 2013 , the $10^{\text {th }} \mathrm{HR}$ showed increased growth, similar to that of the $17^{\text {th }} \mathrm{HR}(p>0.05)$ and higher than that of the $9^{\text {th }} \mathrm{HR}(p<0.05)$. Conclusion: The Implementation Index proved to be adequate to evaluate the performance indicators of the Rede Mãe Paranaense healthcare network in the three Health Regionals analyzed.

Keywords: program evaluation; health systems; women's health; child health.

\section{INTRODUÇÃO}

Os programas ou ações programáticas em saúde na área materno-infantil sempre tiveram, historicamente, um espaço relevante na agenda de compromissos dos governantes no mundo. No Brasil, desde a década de 1940 até a atualidade, várias estratégias sociais, econômicas e em saúde foram criadas no intuito de melhorar a qualidade de vida de mulheres e crianças, que, por conseguinte, colaboraram significativamente para a mudança das taxas de fertilidade, de morbidade e de mortalidade'. Tais mudanças ocorreram com maior ênfase a partir da década de 1980, com a criação do Sistema Único de Saúde (SUS) e, posteriormente, com a reorganização dos serviços de saúde em diferentes níveis de atenção (primário, secundário e terciário), sendo o primário considerado a porta de entrada desse sistema ${ }^{2,3}$.

O Programa Saúde da Família (PSF), lançado em 1994, foi fundamental para apoiar a assistência à gestante e à criança na Atenção Primária à Saúde (APS). Iniciou-se a construção de uma atenção em saúde diferenciada das anteriores, com foco em ações de prevenção e na promoção da saúde. Em 2000, com a Declaração do Milênio pela Organização das Nações Unidas (ONU), o Ministério da Saúde (MS) implementou o Programa de Humanização no Pré-Natal e Nascimento (PHPN) e o Sistema de Acompanhamento da Gestante (Sisprenatal), visando melhorar a qualidade das informações para planejamento, acompanhamento e avaliação das ações ${ }^{2,4}$.

Por conseguinte, quase duas décadas após a Declaração do Milênio, o MS reorganizou as ações de saúde materno-infantil por meio das Redes de Atenção à Saúde (RAS) e lançou a Rede Cegonha, no intuito de assegurar às mulheres o direito ao planejamento reprodutivo, à atenção humanizada à gravidez, ao parto e ao puerpério, bem como às crianças o direito ao nascimento seguro, ao crescimento e ao desenvolvimento saudável, além de reduzir a mortalidade desse grupo populacional, com ênfase no componente neonatal ${ }^{5}$. A implantação dessa Rede no Paraná deu-se com a criação da Rede Mãe Paranaense (RMP), em 2012, fundamentada nos princípios gerais da Rede Cegonha ${ }^{6,7,8}$, bem como nas experiências exitosas do estado, com o Programa Mãe Curitibana, em 1999, o qual apresentou resultados favoráveis, com uma proposta de melhoria da qualidade do pré-natal e garantia de acesso ao parto, consulta puerperal precoce, visitas à detecção e manejo de possíveis complicações e estímulo ao aleitamento materno. Também com o Programa de Qualificação da Atenção Primária à Saúde (APSUS), em 2011, para a educação permanente, houve investimentos de infraestrutura, custeio das equipes e implantação das RAS: Mãe Paranaense, Urgência e Emergência, Pessoa com Deficiência, Saúde Mental e Pessoa Idosa ${ }^{6,8}$.

As Redes podem ser caracterizadas pelos seguintes aspectos: interdependência, autonomia, confiança, cooperação e relações relativamente estáveis ${ }^{6}$. A portaria que instituiu as RAS define Rede como arranjos organizativos de ações e serviços de saúde, de diferentes densidades tecnológicas, que, integradas por meio de sistemas de apoio técnico, logístico e de gestão, buscam garantir a integralidade do cuidado ${ }^{5-7}$. A APS tem por função ser a base das Redes, sendo resolutiva, coordenando o cuidado e ordenando-as ${ }^{7}$.

A assistência materno-infantil em Rede, tanto o Cegonha quanto o Mãe Paranaense ${ }^{5,8}$, prevê ações para: garantia do acolhimento com avaliação e classificação de risco e vulnerabilidade; ampliação do acesso e melhoria da qualidade do pré-natal; garantia de vinculação da gestante à unidade de referência e ao transporte seguro; garantia das boas práticas e segurança na 
atenção ao parto e nascimento; garantia da atenção à saúde das crianças de 0 a 24 meses com qualidade e resolutividade; e garantia de acesso às ações do planejamento reprodutivo ${ }^{5}$.

Embora muitos programas destinados à população materno-infantil tenham sido implementados ao longo das décadas, a avaliação nem sempre foi tão necessária como nos tempos atuais, por causa da complexidade do sistema de saúde e de suas interfaces com outros setores, bem como por ser considerada uma ferramenta fundamental para os gestores obterem informações quanto ao impacto das ações e responderem se as escolhas atenderam aos anseios da população'.

A importância da avaliação de programas e serviços de saúde no contexto brasileiro vem ocorrendo em diversos momentos, no intuito de inserir a prática da avaliação no planejamento e na implementação de programas e de ações ${ }^{10}$. A avaliação também visa analisar os efeitos ou os impactos gerados por uma intervenção, a fim de que decisões sejam tomadas quanto à reestruturação ou à finalização ${ }^{11,12}$. Donabedian ${ }^{13}$ afirma que a avaliação de programas deve ser fundamentada em um tripé: estrutura, processo e resultados.

Especificamente, a avaliação dos resultados poderá identificar o quanto a Rede foi capaz de evitar, minimizar ou mesmo interromper a evolução do processo saúde-doença. A abordagem quantitativa descreve, de forma detalhada, a relação funcional dos serviços e, principalmente, dos mecanismos de comunicação e de operação entre os diferentes serviços da Rede, permitindo identificar as variáveis de funcionamento e as organizações individual e coletiva ${ }^{10}$. Após a implementação do PHPN, diferentes estudos nacionais propuseram indicadores avaliativos, tornando-se crescente a utilização de parâmetros de avaliação ${ }^{14}$.

Considerando que a avaliação pode nortear a tomada de decisão dos gestores quanto aos programas e às ações desenvolvidas nos municípios e estados brasileiros, e que há ausência de pesquisas sobre a implantação dessas Redes, o presente estudo teve como objetivo analisar o Índice de Implementação (II) antes e depois da RMP em três Regionais de Saúde (RS).

\section{MÉTODO}

Trata-se de uma pesquisa quantitativa retrospectiva realizada em três RS do estado do Paraná, Brasil, sendo elas: $9^{a}$ RS de Foz do Iguaçu, constituída de 9 municípios; 10a RS de Cascavel, formada por 25 municípios; e 17a RS de Londrina, composta por 21, totalizando 55 municípios.

O período de estudo compreendeu os anos de 2010 a 2011, antes da implantação da RMP, e de 2012 a 2013, depois da implantação. A coleta foi realizada de maio a outubro de 2014. As fontes de dados utilizadas para construir os indicadores de avaliação da implementação da RMP foram: o Sisprenatal, o Sistema de Informações sobre Nascidos Vivos (Sinasc), o Sistema de Informações do Programa Nacional de Imunizações (SI-PNI), o Sistema de Informações sobre Mortalidade (SIM) e algumas informações que estavam disponíveis somente nas RS.

A construção do instrumento de coleta de dados contemplou os pressupostos estabelecidos pela linha guia da $\mathrm{RMP}^{8}$, composto por 10 questões distribuídas em 22 variáveis: 1) $n^{\circ}$ de gestantes cadastradas no Sisprenatal (Fonte: RS) $n^{\circ}$ de gestantes, gestantes com $\geq$ 7 consultas, $n^{\circ}$ total de consultas, $n^{\circ}$ de gestantes que iniciaram o pré-natal no $1^{\circ}$ trimestre $e$ $\mathrm{n}^{\circ}$ de consultas odontológicas; 2 ) quantidade de exames realizados por trimestre gestacional (Fonte: $\mathrm{RS})-1{ }^{\circ}, 2^{\circ}$ e $3^{\circ}$ trimestres, teste rápido para gravidez, sífilis e HIV; 3 ) estratificação de risco gestacional (Fonte: $\mathrm{RS}$ ) - implantação da estratificação de risco habitual (RH), intermediário (RI) e alto risco (AR) e atendimento ambulatorial especializado para RI e AR; 4) contratualização dos hospitais para a vinculação do parto conforme a estratificação de risco (Fonte: RS); 5) incentivo de qualidade ao parto ( $\mathrm{n}^{\circ}$ e tipos) (Fonte: Sinasc; RS); 6) mortalidade materna (Fonte: SIM); 7 ) $n^{\circ}$ de nascidos vivos (Fonte: Sinasc); 8 ) mortalidade infantil (mortalidade neonatal precoce MNP; mortalidade neonatal tardia - MNT; e mortalidade pós-neonatal - MPN) (Fonte: SIM); 9) presença de Comitês de Investigação de Mortalidade Materna e Infantil (CIMMI) (Fonte: RS); 10) cobertura vacinal em $\leq 1$ ano de idade (Fonte: $\mathrm{SI}-\mathrm{PNI}$ ) - hepatite B recombinante, adsorvida difteria, tétano, pertussis, hepatite $B$ recombinante e Haemophilus influenzae $b$ conjugada (penta), poliomielite 1, 2 e 3 inativada (VIP) e poliomielite 1, 2 e 3 atenuada (VOP), BCG, sarampo, caxumba, rubéola (tríplice viral), febre amarela atenuada (FA), rotavírus humano G1P1[8] 
atenuado (VORH), meningocócica C conjugada, pneumocócica conjugada 10 valente (pneumo 10). Lembrando que a penta foi introduzida em 2012; sendo assim, foi avaliada a tetravalente até 2012 e, posteriormente, a penta em 2013. A tetra viral foi introduzida no calendário vacinal em setembro de 2013, também sendo contabilizada.

A partir das variáveis, estabeleceram-se cinco domínios a serem avaliados: 1) consultas $n^{\circ}$ total de gestantes, gestantes com $\geq 7$ consultas, $n^{\circ}$ total de consultas, $n^{\circ}$ de gestantes que iniciaram o pré-natal no $1^{\circ}$ trimestre e $n^{\circ}$ de consultas odontológicas; 2 ) prevenção materna - $n^{\circ}$ de exames nos $1^{\circ}, 2^{\circ}$ e $3^{\circ}$ trimestres, teste rápido de sífilis, gravidez e HIV; 3 ) risco - estratificação de gestantes (RA, RI e AR), atendimento ambulatorial especializado para RI e AR e tipo de parto; 4) gestão - termo de contrato para gestação de $R H, R I$ e $A R$; 5) seguimento - mortalidade materna, mortalidade infantil (MNP, MNT e MPN), CIMMI e vacinas.

Após agrupamento dos domínios, realizou-se a somatória dos pesos previamente estabelecidos e normatizados em escala de porcentagem (\%), categorizados em dois níveis: 0 = informação inexistente; e 1 = informação existente nas bases de dados. Apenas para a variável "parto" adotaram-se: 0 = mais de $15 \%$ de partos cesáreos registrados; e 1 = até $15 \%{ }^{8}$ (Equação 1).

$$
\text { Índice de Implementação }(I I)=\frac{\text { Consultas }+ \text { Prevenção Materna }+ \text { Risco }+\frac{\text { Gestão }}{\text { Vinculação }}+\text { Seguimento }}{5}
$$

Para avaliar quais indicadores influenciaram o processo de implementação da RMP, para o II, utilizou-se do teste de Shapiro-Wilk para avaliar o padrão de distribuição e, conforme tendiam à homogeneidade das variâncias por meio do teste de Levene, realizou-se a modelagem, assumindo o Il como variável dependente e os indicadores como variáveis independentes. Em seguida, os dados relativos à classificação de adequação à RMP (adequado e inadequado) foram relacionados aos indicadores considerados estatisticamente significativos nas análises estatísticas univariadas, por meio da análise de regressão logística.

$\mathrm{Na}$ análise de regressão logística, utilizou-se do modelo Logit, método Stepwise Forward, com tipo de resposta binária. Para tal, algumas etapas foram cumpridas:

a) seleção das variáveis usando critério de $p<0,20$ da razão de chance (odds ratio) ser estatisticamente equivalente a 1,00;

b) obtenção de um modelo com número reduzido de variáveis, selecionadas a partir da aplicação do critério de $p<0,10$ da razão de chance ajustada ser estatisticamente equivalente a 1,00;

c) obtenção de um modelo final após teste para todas as interações múltiplas possíveis, usando a maximização da função de verossimilhança com o algoritmo de Newton-Raphson.

Ao decidir pelo modelo final de regressão logística, as probabilidades foram calculadas a partir da fórmula $\operatorname{Pr}=1 /[1+e-(a+\Sigma(\beta i$ xi $)]$, sendo a a constante do modelo. A análise foi finalizada com o cálculo da probabilidade de risco, pela estimativa da probabilidade de ocorrência de risco de inadequação do município à RMP, analisando a combinação das variáveis expostas no modelo preditivo.

Em seguida, aplicou-se o modelo linear de análise da variância fatorial, método dos quadrados do tipo I, com seleção do modelo pelo método Stepwise. A probabilidade de entrada para o modelo foi de 0,05 , e a de saída, igual a 0,10.

Para o ajuste do modelo, empregou-se o critério de Akaike (AIC). Após a definição do modelo de predição, o II foi avaliado entre as RS ao longo dos quatro anos (de 2010 a 2011 e de 2012 a 2013). Para tal, aplicou-se o teste Anova para medidas repetidas, considerando as RS como fator independente.

As variáveis que foram consideradas significativas pelo modelo ajustado foram comparadas entre as RS por meio do método de Monte Carlo. Em todas as análises, assumiu-se um nível de significância de $p<0,05$, utilizando-se do programa XLStat2014.

A pesquisa foi aprovada pelo Comitê de Ética em Pesquisa da Universidade Estadual do Oeste do Paraná, Parecer nº 544.107/2014, CAAE: 26317614.8.1001.0107. 


\section{RESULTADOS}

Após a análise do Il da RMP nas três RS em estudo, de 2010 a 2013, foi possível identificar a influência de variação do II $\left(F_{18,201}=31085,179 ; p<0,0001\right)$ (Tabela 1).

Na Tabela 1, também se identifica que, após a aplicação do modelo ajustado, foi possível verificar as variáveis que influenciam positivamente (presença de informação $=1$ ) e negativamente (ausência de informação $=0$ ) o Il da RMP.

Pode-se observar que a presença ou a disponibilidade de informações sobre o $n^{\circ}$ de gestantes cadastradas com $\geq 7$ consultas, o $n^{\circ}$ de gestantes que iniciaram o pré-natal no $1{ }^{\circ}$ trimestre, a classificação das gestantes de $\mathrm{RH}$, a contratualização dos atendimentos às gestantes de $\mathrm{RH}$ e $\mathrm{AR}$, a vacinação adequada entre $\leq 1$ ano de idade para $\mathrm{BCG}$, tríplice viral,

Tabela 1. Variáveis com indicadores positivos e negativos que influenciaram o Índice de Implementação da Rede Mãe Paranaense, conforme os parâmetros do modelo ajustado, 9a 10ª e 17a Regionais de Saúde, de 2010 a 2013

\begin{tabular}{|c|c|c|c|c|c|c|}
\hline $\begin{array}{l}\text { Variáveis com indicadores } \\
\text { positivos (1) e negativos (0) }\end{array}$ & Valor & $\begin{array}{l}\text { Erro } \\
\text { padrão }\end{array}$ & $t$ & $\operatorname{Pr}>|\mathbf{t}|$ & $\begin{array}{l}\text { Limite } \\
\text { inferior } \\
(95 \%)\end{array}$ & $\begin{array}{c}\text { Limite } \\
\text { superior } \\
(95 \%)\end{array}$ \\
\hline Intercepto & 66,943 & 0,827 & 80,939 & $<0,0001$ & 65,312 & 68,574 \\
\hline \multicolumn{7}{|l|}{ Domínio 1: consultas (nº) } \\
\hline Com $\geq 7$ (1) & 4,197 & 0,294 & 14,278 & $<0,0001$ & 3,617 & 4,776 \\
\hline $1^{\circ}$ trimestre $(1)$ & 5,370 & 0,135 & 39,751 & $<0,0001$ & 5,104 & 5,637 \\
\hline Odontológicas (0) & $-5,145$ & 0,110 & $-46,567$ & $<0,0001$ & $-5,363$ & $-4,928$ \\
\hline $\mathrm{N}^{\circ}$ consultas/ $\mathrm{n}^{\circ}$ gestantes $(0)$ & $-5,382$ & 0,317 & $-16,976$ & $<0,0001$ & $-6,007$ & $-4,756$ \\
\hline \multicolumn{7}{|l|}{ Domínio 2: prevenção materna } \\
\hline Exames no $1^{\circ}$ trimestre $(0)$ & 20,067 & 0,104 & 193,231 & $<0,0001$ & $-20,272$ & $-19,863$ \\
\hline \multicolumn{7}{|l|}{ Domínio 3: risco } \\
\hline Atendimento ambulatorial (0) & $-3,337$ & 0,314 & $-10,625$ & $<0,0001$ & $-3,956$ & $-2,718$ \\
\hline Classificação RH (1) & 4,178 & 0,162 & 25,817 & $<0,0001$ & 3,859 & 4,497 \\
\hline Classificação RI (0) & $-5,269$ & 0,142 & $-37,218$ & $<0,0001$ & $-5,548$ & $-4,990$ \\
\hline Classificação AR (0) & $-1,699$ & 0,278 & $-6,119$ & $<0,0001$ & $-2,247$ & $-1,151$ \\
\hline № parto cesáreo > 15\% (0) & $-3,454$ & 0,093 & $-37,136$ & $<0,0001$ & $-3,637$ & $-3,270$ \\
\hline \multicolumn{7}{|l|}{ Domínio 4: gestão } \\
\hline Contrato gestação RH (1) & 5,538 & 0,173 & 32,069 & $<0,0001$ & 5,198 & 5,879 \\
\hline Contrato gestação RI (0) & $-4,670$ & 0,147 & $-31,879$ & $<0,0001$ & $-4,959$ & $-4,382$ \\
\hline Contrato gestação AR (1) & 8,234 & 0,179 & 46,061 & $<0,0001$ & 7,882 & 8,587 \\
\hline \multicolumn{7}{|l|}{ Domínio 5: seguimento } \\
\hline CIMMI (0) & $-1,658$ & 0,136 & $-12,213$ & $<0,0001$ & $-1,925$ & $-1,390$ \\
\hline Vacinação: BCG (1) & 1,523 & 0,400 & 3,804 & 0,0000 & 0,734 & 2,313 \\
\hline Vacinação: tríplice viral (1) & 1,620 & 0,406 & 3,986 & $<0,0001$ & 0,819 & 2,422 \\
\hline Vacinação: antiamarílica (1) & 1,620 & 0,406 & 3,986 & $<0,0001$ & 0,819 & 2,422 \\
\hline Vacinação: meningocócica (1) & 1,258 & 0,113 & 11,090 & $<0,0001$ & 1,034 & 1,482 \\
\hline
\end{tabular}

PN: pré-natal; RH: risco habitual; Rl: risco intermediário; AR: alto risco 
FA e meningo $C$ influenciaram nos quatro anos em estudo, ou seja, municípios que cumpriam tais metas tiveram maior êxito no processo de implementação da RMP.

Ao contrário, a ausência de informações sobre $n^{\circ}$ consultas odontológicas realizadas às gestantes, $n^{\circ}$ de consultas pelo $n^{\circ}$ de gestantes, $n^{\circ}$ de gestantes com exames no $1^{\circ}$ trimestre, atendimento ambulatorial, classificação de risco das gestantes (RI e AR), no de partos cesáreos, contratualização de atendimento às gestantes de RI e n de CIMMI influenciou negativamente a implementação da RMP nos municípios das respectivas RS.

$\mathrm{O} \mathrm{n}^{\circ}$ de exames realizados no $2^{\circ}$ e $3^{\circ}$ trimestres, o teste rápido para gravidez, sífilis e HIV, o $\mathrm{n}^{\circ}$ e tipos de partos, a mortalidade materna, o $\mathrm{n}^{\circ}$ de nascidos vivos, a mortalidade infantil (MNP, MNT e MPN), o $n^{\circ}$ absoluto de doses de vacinas aplicadas em $\leq 1$ ano de idade para as vacinas de hepatite $B$ (recombinante), penta, VIP e VOP, VORH e pneumo 10 não apresentaram influência estatisticamente significante no II.

Ao realizar a avaliação do II antes (2010 a 2011) e depois (2012 a 2013) da RMP nas 9a, $10^{a}$ e $17^{a}$ RS, foi possível verificar que houve diferenças estatísticas significativas $\left(F_{6,156}=129,14\right.$; $\mathrm{p}=0,000$ ) (Figura 1).

Na 9a RS, ocorreu crescimento significativo de suas médias até o ano de $2012(p<0,05)$, mantendo-se com a mesma pontuação média em 2013, quando comparada ao ano anterior ( $p$ $>0,05)$. Na $10^{\mathrm{a}} \mathrm{RS}$, houve crescimento significativo ao longo dos quatro anos $(p<0,05)$, contudo as médias entre 2011 e 2012 foram consideradas estatisticamente equivalentes $(p>0,05)$. Já na $17^{a} \mathrm{RS}$, o crescimento das médias foi estatisticamente significativo em todos os anos $(p<0,05)$.

Ao realizar comparações inter-regionais, identificou-se que, em 2010, todas as RS apresentaram médias estatisticamente semelhantes $(p>0,05)$, demonstrando a equivalência de todos os municípios. De 2010 a 2011, a 10 RS apresentou uma melhora do II maior que a $9^{a} \mathrm{RS}$. A partir de então, a 9a RS permaneceu com valores do ll sempre menores que as outras regionais avaliadas.

De 2011 a 2012, os valores observados na $10^{\text {a }}$ e na $17^{\text {a }}$ RS foram considerados estatisticamente superiores aos da $9^{\text {a }} \mathrm{RS}(p<0,05)$, entretanto a $10^{\text {a }} \mathrm{RS}$ foi a única que não

$F(6,156)=129,14, p=0,0000$

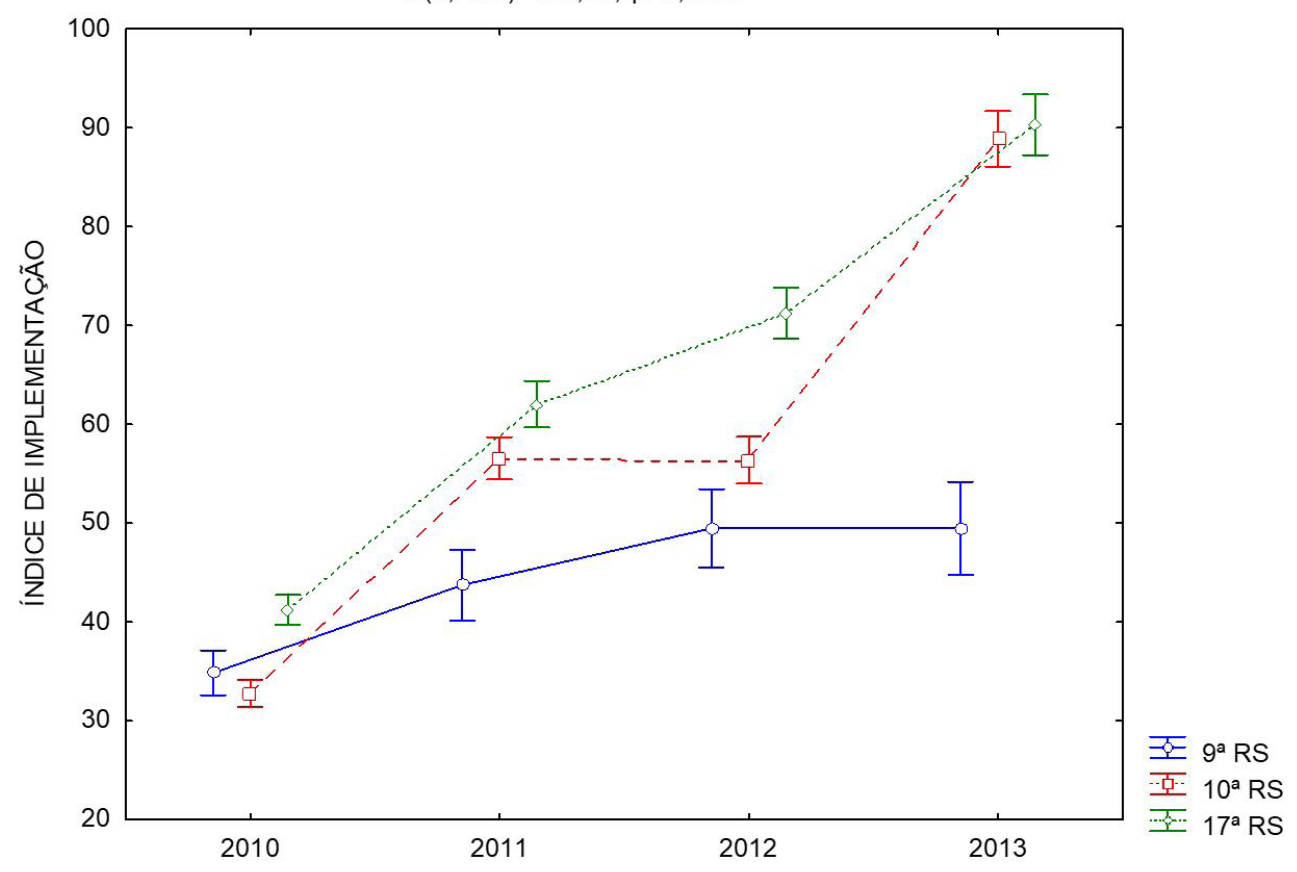

Figura 1. Médias e intervalos de confiança do Índice de Implementação antes (2010-2011) e depois (20122013) da Rede Mãe Paranaense, $9^{a}, 10^{\text {a }}$ e $17^{a}$ Regionais de Saúde 
apresentou melhora do II em todo o ano. De 2012 a 2013, verificou-se que a 10a RS teve elevado crescimento, voltando a apresentar valores estatisticamente semelhantes aos da $17^{\text {a }}$ RS $(p>0,05)$ e superiores aos valores observados na $9^{a} \mathrm{RS}(p<0,05)$, sendo que, nesse período, foi a 9a RS que não teve melhora do II.

\section{DISCUSSÃO}

Para que a avaliação subsidie a gestão em saúde identificando os problemas e mensurando o impacto dos programas implementados, faz-se necessária a escolha de indicadores fidedignos, bem como o adequado ajuste e a predição do modelo utilizado, pois a ausência da definição de critérios pode resultar no insucesso avaliativo. No presente estudo, após a definição dos indicadores e a aplicação do método estatístico, verificou-se que a escolha do modelo foi pertinente para avaliar o ll das práticas/metas definidas na RMP nas três RS. Mas as bases de dados nacionais, em especial as regionais, não ofereceram informações em sua totalidade ${ }^{15,16}$. Embora a completude e a confiabilidade dos sistemas nacionais de informação em saúde tenham atingido maior qualidade nas últimas décadas ${ }^{1}$, ainda assim os estaduais/ regionais continuam tendo limitações e precisam ser superados.

No que se refere ao Sisprenatal, diversas mudanças têm sido realizadas para melhorar o programa, porém estudos demonstram que existem falhas no software do sistema e que a capacidade de processamento inapropriado desencadeia lentidão dos processos de trabalho, agravando a sobrecarga de serviço ${ }^{17-19}$. Ressalta-se que a dificuldade no uso desse sistema engloba questões técnicas e profissionais, gerando subnotificação e repercutindo no repasse dos recursos para os municípios ${ }^{17,18}$.

No Brasil, o número de consultas pré-natais vem aumentando, mas está aquém das metas ministeriais. Consoante a essa realidade, a RMP determina a realização de sete consultas, seis no pré-natal e uma no puerpério ${ }^{8}$, o que, neste estudo, influenciou o Il.

Entre 2005 e 2015, houve aumento da cobertura e qualidade da atenção pré-natal em quase todo o país, variando de 4,5 a 66,1\% em várias regiões brasileiras ${ }^{20}$. Mas em Vitória, Espírito Santo, nenhuma gestante teve um processo de assistência pré-natal totalmente adequado aos critérios estabelecidos pelo PHPN e pela Organização Mundial de Saúde ${ }^{21}$.

O registro dos exames laboratoriais no $1^{\circ}$ trimestre foi inexistente no presente estudo, bem como o registro do número de consultas odontológicas, resultado semelhante ao de João Pessoa, Paraíba ${ }^{16}$. Entretanto, pré-natal classificado como adequado foi o que apresentou maior frequência de registro ${ }^{22,23}$.

A estratificação da gestação de RH constava no sistema de informação das RS. A RMP utiliza a estratificação de risco gestacional em RH e AR, conforme a Rede Cegonha ${ }^{5}$, incrementando o $\mathrm{Rl}$, que abrange características individuais da gestante (raça, etnia e idade), sociodemográficas (escolaridade) e de história reprodutiva anterior ${ }^{8}$.

Nas três RS, o parto cirúrgico superou os $15 \%$, evidenciando que é uma realidade semelhante ao do país ${ }^{4}$ que ocupa a segunda posição no ranking mundial. Na rede privada, esse tipo de parto atingiu $80 \%$, e, na pública, $40 \%$, entre 2000 e 2010. A taxa de parto cirúrgico, no mesmo período, foi de 31,8\% nos Estados Unidos, 20,2\% na França e 22,7\% na Argentina ${ }^{5,24}$.

Após o nascimento, no seguimento do serviço de saúde, as vacinações (BCG, tríplice viral, FA e meningocócica C) influenciaram o II, ao contrário da hepatite B (recombinante), pentavalente, VIP e VOP, VORH e pneumocócica 10. Diferentemente deste estudo, uma pesquisa evidenciou que os registros nos sistemas de informação se mostraram o melhor estimador a ser utilizado para identificar a cobertura vacinal ${ }^{25}$.

Quanto à informação sobre o número de CIMMI nas três RS, verificou-se que ela esteve ausente no presente estudo, mesmo que a sua implementação tenha sido uma normativa do MS, o qual determina que, em cidades com mais de 80 mil habitantes, deve haver um Comitê Municipal, ou na Regional, um Comitê Estadual. Ressalta-se que a investigação da causa básica de morte oferece subsídios para os gestores implementarem ações preventivas e reduzirem as mortes materno-infantis consideradas evitáveis. 
Ao analisar o II da RMP, verifica-se que, embora a ausência de informações tenha sido uma realidade, ficou evidente o impacto positivo após a implementação da RMP nas três RS, com ênfase em uma delas, a 17a RS. Sobre esse resultado, infere-se, possivelmente, a melhor organização do sistema de saúde na prestação de cuidado materno-infantil, mas há muito ainda a se fazer para garantir a integralidade da assistência e alcançar as metas nacionais e internacionais.

Entre os desafios a serem superados, não somente nas regionais em estudo, está tornar as ações em Redes que buscam, a partir dos pontos de atenção e diagnósticos, gerenciar os diferentes níveis de complexidade distribuídos em um território, prestando cuidado coordenado pela APS no tempo e no lugar, com o custo e a qualidade certos e humanizados, mediante as responsabilidades sanitárias e econômicas ${ }^{7}$.

As ações de assistência materno-infantil em Rede dispõem de quatro componentes estruturantes ${ }^{5}$. O primeiro se refere ao pré-natal, mediante cuidado qualificado e prestado na atenção primária, com captação precoce das gestantes, acolhimento às intercorrências gestacionais, por meio da avaliação e da classificação de risco e vulnerabilidade, acesso ao pré-natal de AR em tempo oportuno, realização de exames, vinculação da gestante ao local em que será realizado o parto, qualificação do sistema de informação, implementação de ações socioeducativas relacionadas à educação sexual/reprodutiva, prevenção e tratamento das IST/ HIV/Aids, sífilis, hepatites e apoio ao deslocamento das gestantes.

O segundo diz respeito ao âmbito hospitalar para o parto e nascimento, que deve oferecer leitos obstétricos e neonatais, ambiência das maternidades conforme a legislação da Agência Nacional de Vigilância Sanitária (Anvisa), práticas de atenção à saúde baseada em evidências científicas, garantia de acompanhante durante todo o processo do pré-parto, parto e pós-parto imediato, realização de acolhimento e classificação de risco, estímulo à implantação de equipes horizontais de cuidado obstétrico e implementação de colegiado gestor nas maternidades, conforme a Política Nacional de Humanização.

O terceiro componente se refere ao seguimento da mulher e criança após a alta da maternidade, no intuito de promover o aleitamento materno e a alimentação complementar saudável, visita domiciliar ao binômio na primeira semana após o parto e nascimento, busca ativa de crianças vulneráveis, promoção da saúde sexual e reprodutiva, prevenção às ISTs e tratamento delas, orientação e oferta de métodos contraceptivos.

O último componente estruturante trata da regulação para promover acesso às urgências por meio do Sistema de Atendimento Móvel de Urgência, sendo que as ambulâncias do sistema avançado devem possuir incubadoras e ventiladores neonatais, implantação e/ou implementação da regulação de leitos obstétricos e neonatais, assim como regulação de urgências e serviços ambulatoriais.

À medida que os gestores cumprem esses componentes assistenciais, eles promovem a integração sistêmica de ações e serviços de saúde com provisão de atenção contínua, integral e de qualidade, reduzem a fragmentação, melhoram a eficiência global do sistema, respondendo às necessidades e expectativas das usuárias ${ }^{5,7}, \mathrm{e}$, consequentemente, reduzem os indicadores que ainda permanecem altos no país, tais como a mortalidade materna e neonatal, em especial nas regiões com maiores desigualdades sociais e em saúde.

As bases de dados nacionais e regionais têm sido amplamente utilizadas para oferecer subsídios avaliativos quanto ao impacto das ações em saúde por causa da agilidade, do acesso e do baixo custo ${ }^{2,9,10}$. Tais informações podem diminuir os obstáculos do processo avaliativo para a implementação de programas e oferecer subsídios na tomada de decisões imediatas, além de, em tempo hábil, reorganizar as ações em saúde ${ }^{26}$.

A definição do Il para avaliar o desempenho da RMP, após ser instituída nas três RS, mostrou-se adequada a cumprir a proposta avaliativa. Embora tenha sido identificada a subnotificação de informações, em especial no Sisprenatal e na base regional, foi possível observar o impacto positivo.

Alguns ajustes ainda devem ser realizados, principalmente quanto ao sistema de informação da estratificação de risco de gestantes e de recém-nascidos, para a vinculação adequada de referência e de contrarreferência entre os níveis de atenção à saúde, melhorando, 
dessa forma, o acesso ágil e de qualidade à assistência materno-infantil, o que reduziria agravos e mortes evitáveis relacionadas à organização desse sistema.

\section{REFERÊNCIAS}

1. Aquino EML, Leal MC, Monteiro CA, Barros FC, Szwarcwald CL. Saúde de mães e crianças no Brasil: progressos e desafios. Lancet. 2011;377(9780):1863-76. PMid:21561656.

2. Cassiano ACM, Carlucci SEM, Gomes CF, Bennemann RM. Saúde materno infantil no Brasil: evolução e programas desenvolvidos pelo Ministério da Saúde. Rev Serviço Público. 2014;65(2):227-44. http://dx.doi. org/10.21874/rsp.v65i2.581.

3. Paim J, Travassos C, Almeida C, Bahia L, Macinko J. O sistema de saúde brasileiro: história, avanços e desafios. Lancet. 2011;377(9779):11781-97. PMid:21561655.

4. Organização Mundial de Saúde. Declaração do Milênio [Internet]. Nova lorque: OMS; 2000 [citado em 2016 fev 4]. Disponível em: https://www.unric.org/html/portuguese/uninfo/DecdoMil.pdf.

5. Brasil. Ministério da Saúde. Manual prático para implementação da Rede Cegonha [Internet]. Brasília: Ministério da Saúde. 2011. [citado em 2015 dez 20]. Disponível em: www.saude.mt.gov.br/arquivo/3062

6. Huçulak MC, Peterlini OLG. Rede Mãe Paranaense: relato de experiência. Rev Saúde Pública Paraná. 2014;15(1):77-86.

7. Mendes EV. As redes de atenção à saúde [Internet]. Brasília: Organização Pan-Americana da Saúde; 2011 549 p. [citado em 2015 dez 20]. Disponível em: http://www.paho.org/bra/index.php?option=com_ docman\&view=download\&alias=1402-as-redes-atencao-a-saude-2a-edicao-2\&category_slug=servicossaude-095\&Itemid=965

8. Paraná. Secretaria de Estado da Saúde do Paraná (SESA-PR). Linha guia: programa Rede Mãe Paranaense [Internet]. Curitiba: SESA-PR; 2018. [citado em 2018 dez 12]. Disponível em: http://www.saude.pr.gov.br/ arquivos/File/ACS/linha_guia_versao_final.pdf

9. Champagne F, Contandriopoulos AP, Brouselle A, Hartz Z, Denis JL. A avaliação no campo da saúde. In: Brousselle A, Champagne F, Contandriopoulos A, Hartz Z, editores. Avaliação: Conceitos e métodos. Rio de Janeiro: Editora Fiocruz; 2011. p. 41-60.

10. Tanaka OY. Avaliação da Atenção Básica em Saúde: uma nova proposta. Saude Soc. 2011;20(4):927-34. http://dx.doi.org/10.1590/S0104-12902011000400010.

11. Brouselle A, Champagne F, Contandriopoulos AP, Hartz Z. Avaliação: conceitos e métodos. Rio de Janeiro: Fiocruz; 2011. 291 p.

12. Figueiró AC, Frias PG, Navarro LM. Avaliação em saúde: conceitos básicos para a prática nas instituições. In: Samico I, Felisberto E, Figueiró AC, Frias PG, editores. Avaliação em saúde: bases conceituais e operacionais. Rio de Janeiro: MedBook; 2010. p. 1-13.

13. Donabedian A. Evaluating the quality of medical care. 1966. Milbank Q. 2005;83(4):691-729. http://dx.doi. org/10.1111/j.1468-0009.2005.00397.x. PMid:16279964.

14. Domingues RMSM, Hartz ZMA, Dias MAB, Leal MC. Avaliação da adequação da assistência pré-natal na rede SUS do Município do Rio de Janeiro, Brasil. Cad Saude Publica. 2012;28(3):425-37. http://dx.doi. org/10.1590/S0102-311X2012000300003. PMid:22415175.

15. Corrêa MD, Tsunechiro MA, Lima MOP, Bonadio IC. Evaluation of prenatal care in unit with family health strategy. Rev Esc Enferm USP. 2014;48(spe.):23-31. http://dx.doi.org/10.1590/S0080-623420140000600004.

16. Silva RS, Oliveira CM, Ferreira DKS, Bonfim CV. Avaliação da completitude das variáveis do Sistema de Informações sobre Nascidos Vivos - Sinasc - nos Estados da região Nordeste do Brasil, 2000 e 2009. Epidemiol Serv Saude. 2013;22(2):347-52. http://dx.doi.org/10.5123/S1679-49742013000200016.

17. Lima AP, Corrêa ACP. A produção de dados e informações para o SISPRENATAL no nível central de gestão. Cienc Cuid Saude. 2012;11(2):352-9. http://dx.doi.org/10.4025/cienccuidsaude.v11i2.16330.

18. Lima AP, Corrêa ACP. A produção de dados para o Sistema de Informação do Pré-Natal em unidades básicas de saúde. Rev Esc Enferm USP. 2013;47(4):876-83. http://dx.doi.org/10.1590/S0080-623420130000400015. PMid:24310685.

19. Moimaz SAS, Garbin CAS, Garbin AJI, Zina LG, Yarid SD, Francisco KMS. Sistema de Informação Pré-Natal: análise crítica de registros em um município paulista. Rev Bras Enferm. 2010;63(3):385-90. http://dx.doi. org/10.1590/S0034-71672010000300006. PMid:20658071.

20. Nunes JT, Gomes KRO, Rodrigues MTP, Mascarenhas MDM. Qualidade da assistência pré-natal no Brasil: revisão de artigos publicados de 2005 a 2015. Cad Saude Colet. 2016;24(2):252-61. http://dx.doi. org/10.1590/1414-462X201600020171. 
21. Polgliane RBS, Leal MC, Amorim MHC, Zandonade E, Santos ET No. Adequação do processo de assistência pré-natal segundo critérios do Programa de Humanização do Pré-natal e Nascimento e da Organização Mundial de Saúde. Ciênc Saúde Coletiva. 2014;19(7):1999-2010. http://dx.doi.org/10.1590/141381232014197.08622013.

22. Correia LOS, Padilha BM, Vasconcelos SML. Métodos para avaliar a completitude dos dados dos sistemas de informação em saúde do Brasil: uma revisão sistemática. Ciênc Saúde Coletiva. 2014;19(11):4467-78. http://dx.doi.org/10.1590/1413-812320141911.02822013.

23. Santos Neto $E T$, Oliveira $A E$, Zandonade E, Leal MC. Acesso à assistência odontológica no acompanhamento pré-natal. Ciênc Saúde Coletiva. 2012;17(11):3057-68. http://dx.doi.org/10.1590/ S1413-81232012001100022.

24. European Union, Organization for economic Co-operation and Development. Health at a Glance: Europe [Internet] 2010 [citado em 2016 mar 10]. Disponível em: http://dx.doi.org/10.1787/health_glance-2010-en.

25. Teixeira AMS, Mota ELA. Denominadores para o cálculo de coberturas vacinais: um estudo das bases de dados para estimar a população menor de um ano de idade. Epidemiol Serv Saude. 2010;19(3):187-203. http://dx.doi.org/10.5123/S1679-49742010000300002.

26. Tanaka OY, Tamaki EM. O papel da avaliação para a tomada de decisão na gestão de serviços de saúde. Ciênc Saúde Coletiva. 2012;17(4):821-8. 